\title{
The challenge of targeting EGFR: experience with gefitinib in nonsmall cell
} lung cancer

\author{
A.A. Armour and C.L. Watkins
}

ABSTRACT: As the first approved epidermal growth factor receptor (EGFR)-targeted therapy for nonsmall cell lung cancer (NSCLC), the clinical development of gefitinib was complex. Advances in scientific understanding of the target biology during its clinical development enabled the identification of a biomarker to define patients most likely to derive benefit from gefitinib. Initial phase II trials showed clinically meaningful anti-tumour activity in $12-18 \%$ of unselected pretreated patients with advanced NSCLC at the optimum biological dose $(250 \mathrm{mg})$. Subgroup analyses of these and subsequent phase III trials in unselected patients suggested that EGFR mutation and some clinical characteristics associated with a higher incidence of EGFR mutation (Asian ethnicity, adenocarcinoma histology, never-smoking and female sex) were linked with increased response to gefitinib. Consequently, the IRESSA Pan-Asia Study (IPASS) was conducted in never-smokers or former light-smokers in East Asia who had adenocarcinoma of the lung. IPASS showed that EGFR mutation was the strongest predictor of improved progressionfree survival (mutation-positive subgroup hazard ratio (HR) $0.48,95 \% \mathrm{Cl}$ 0.36-0.64 (p $<0.001$, $n=261)$; mutation-negative subgroup HR 2.85, 95\% Cl 2.05-3.98 $(p<0.001, n=176)$; interaction test $\mathbf{p}<0.001$ ) with gefitinib versus carboplatin/paclitaxel as first-line therapy for advanced NSCLC. Important lessons for the development of future personalised medicines are discussed.

KEYWORDS: Epidermal growth factor receptor, gefitinib, nonsmall cell lung cancer, personalised medicine

ung cancer is the most common cause of cancer deaths worldwide [1]. Many cases are not diagnosed until the disease is at an advanced stage, and the prognosis is poor with a 5 -yr survival rate of $\sim 15 \%$ [2]. Nonsmall cell lung cancer (NSCLC) accounts for $\sim 80 \%$ of all lung cancers and comprises three main types: adenocarcinoma, squamous cell carcinoma and large cell carcinoma. Advances have been made in the control of local disease with the addition of systemic therapy; however, for patients with metastatic disease, chemotherapy has remained the established treatment since the mid-1990s [3]. Platinum-based doublet chemotherapy has been the mainstay of first-line treatment for advanced NSCLC in patients with a good performance status; however, despite the development of new chemotherapy regimens, the prognosis remains poor and the toxicity remains significant [4]. Targeted cancer therapies focusing on molecular changes specific to cancer may be more effective, and give rise to predictable and more favourable tolerability, than traditional chemotherapy that interferes with all rapidly dividing cells.

Gefitinib (IRESSA ${ }^{\mathrm{TM}}$ ) is an orally active epidermal growth factor receptor (EGFR)-tyrosine kinase inhibitor (TKI) and was the first EGFR-targeted therapy to be approved for the treatment of NSCLC. The understanding of the biological role of the target/receptor was limited during the early stages of gefitinib development. The clinical programme needed to be adapted in response to advances in scientific understanding of the target biology.

Phase II clinical studies demonstrated antitumour activity of gefitinib against pretreated advanced NSCLC, with response rates of $12-18 \%$ in an unselected population [5,6]. Gefitinib was subsequently approved in Japan in 2002 and in the USA in 2003. The results of the phase III
AFFILIATIONS

AstraZeneca, Macclesfield, UK.

CORRESPONDENCE

A.A. Armour

AstraZeneca

Alderley Park

Macclesfield

Cheshire

SK10 4TG

UK

E-mail: Alison.Armour@

astrazeneca.com

Received:

May 212010

Accepted after revision:

June 292010

PROVENANCE

Publication of this peer-reviewed article was supported by

AstraZeneca, UK (principal sponsor, European Respiratory Review issue 117). 
IRESSA Survival Evaluation in Lung cancer (ISEL) study available in 2004 failed to show a statistically significant benefit in overall survival for gefitinib compared with best supportive care in the overall unselected, predominantly refractory study population [7]. This led to the use of gefitinib being restricted in June 2005 by the US Food and Drug Administration (FDA) and to AstraZeneca withdrawing the marketing authorisation application under review in Europe. Greater benefit for gefitinib versus placebo observed in subgroups such as neversmokers and patients of Asian origin suggested the potential for improved patient selection based on clinical characteristics, which was explored in further work in conjunction with tumour biology studies. In 2008, the phase III IRESSA Pan-Asia Study (IPASS) showed that the presence of an EGFR mutation was the strongest predictor of a more favourable outcome with gefitinib compared with carboplatin/paclitaxel as first-line treatment. In June 2009, the European Medicines Agency (EMA) approved gefitinib for use in adult patients with locally advanced or metastatic NSCLC with activating mutations of EGFR-tyrosine kinase.

This article discusses the complex journey of discovery and clinical development of gefitinib and the many challenges that were successfully resolved. Advances in the scientific understanding of the target biology, which resulted in the ability to biologically define those patients who were most likely to derive the greatest benefit from treatment, are also reviewed.

\section{TARGETING THE EGFR WITH GEFITINIB}

EGFR belongs to a family of four related transmembrane receptors: EGFR (HER1), HER2, HER3 and HER4 [8, 9]. EGFR is activated by binding of one of its specific ligands, such as epidermal growth factor (EGF) or transforming growth factor$\alpha$, to its extracellular domain, resulting in dimerisation, and receptor autophosphorylation and transphosphorylation through intrinsic tyrosine kinase activity. This triggers intracellular pathways that can result in cell proliferation, inhibition of apoptosis, invasion and metastasis, and tumour-induced angiogenesis [10]. Many common solid tumours of epithelial origin express high levels of EGFR and it has been associated with advanced disease and poor prognosis [8]; it is, therefore, an attractive target for anti-tumour therapies.

Gefitinib is a low molecular weight, synthetic anilinoquinazoline that was designed to inhibit the EGFR pathway. Gefitinib inhibits the tyrosine kinase activity of EGFR, blocking its autophosphorylation and subsequent downstream signalling (fig. 1) [12]. In preclinical studies, gefitinib inhibited EGFstimulated cell growth and tumour growth in nude mice bearing a range of human tumour xenografts, illustrating its potential for cancer therapy [12].

\section{DETERMINING THE OPTIMUM BIOLOGICAL DOSE}

The tolerability, pharmacokinetics and anti-tumour activity of oral gefitinib at doses up to $1,000 \mathrm{mg} \cdot \mathrm{day}^{-1}$ were investigated in four phase I dose-escalation multicentre studies in patients with a range of solid tumours known to express EGFR, including 100 patients with advanced NSCLC [13-16]. In two initial phase I studies, gefitinib was administered once daily for 14 days followed by 14 days of observation in 28-day cycles $[15,16]$. The tolerability profile was acceptable and the two subsequent studies were conducted using 28-day cycles of once daily oral dosing at $150-1,000 \mathrm{mg} \cdot$ day $^{-1}$ gefitinib $[13,14]$.

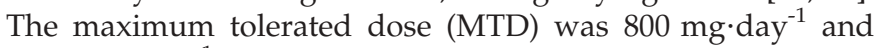
$1,000 \mathrm{mg} \cdot \mathrm{day}^{-1}$ with once daily oral dosing, and the predominant dose-limiting toxicity was diarrhoea $[13,14]$. The most common adverse events were rash/acne, diarrhoea, nausea, vomiting and asthenia, and were dose-related: the majority were mild or moderate (common toxicity criteria (CTC) grade 1 or 2), with grade 3 or 4 events generally occurring at doses

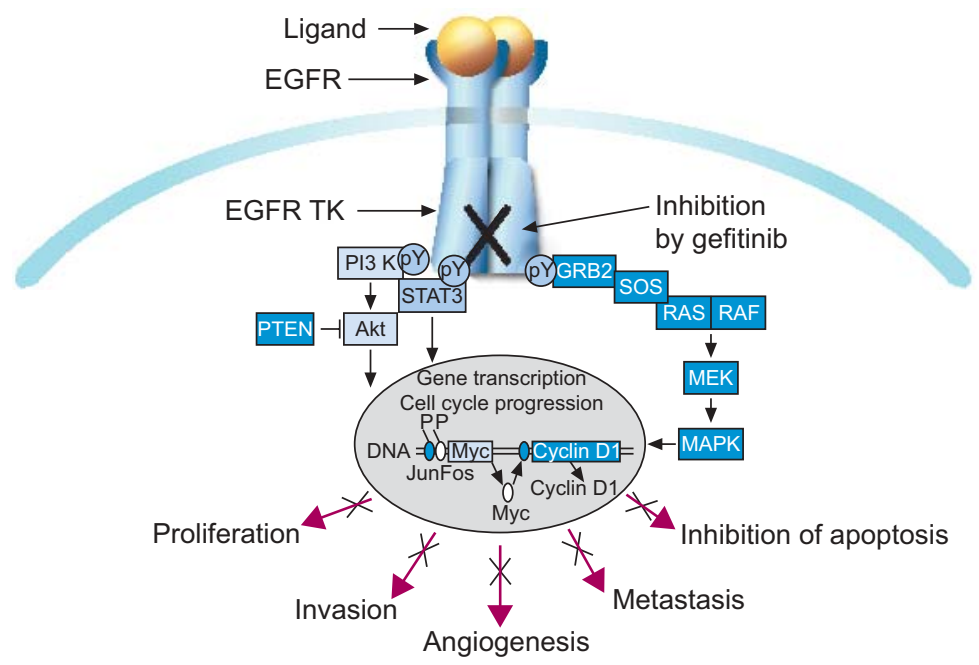

FIGURE 1. Schematic representation of the role of epidermal growth factor receptor (EGFR) in cancer and its inhibition by gefitinib. Activation of the EGFR by ligand binding causes receptor dimerisation and the autophosphorylation of specific tyrosine residues of the intracellular tyrosine kinase (TK) domain. This leads to the stimulation of downstream signalling pathways, including the phosphatidylinositol 3-kinase (PI3K)-Akt and RAS-mitogen-activated protein kinase (MAPK) pathways, which promote the processes of cell proliferation, angiogenesis, invasion/metastasis and the inhibition of apoptosis. Gefitinib inhibits the TK activity of the EGFR TK domain, blocking the signalling pathways important in the survival and proliferation of tumour cells. PTEN: phosphatase and tensin homologue; STAT3: signal transducer and activator of transcription 3; GRB2: growth factor receptor-bound protein 2. Reproduced from [11] with permission from the publisher. 
$>600 \mathrm{mg} \cdot$ day $^{-1}[13,14]$. Radiographic responses were observed across the whole dose range, with all of the 10 partial responses across the trials occurring in patients with NSCLC [13-16].

Dose selection for gefitinib was based on the optimum biological dose approach with the aim of achieving maximum inhibition of the EGFR target at a dose level below the MTD $[17,18]$. In the phase I studies, biologically relevant plasma concentrations of $>100 \mathrm{ng} \cdot \mathrm{mL}^{-1}$ (above the $90 \%$ maximal inhibitory concentration for inhibition of growth of $\mathrm{KB}$ oral carcinoma cells [9]) were generally maintained at doses $>100 \mathrm{mg} \cdot \mathrm{day}^{-1}$ across the 24-h dosing period [15-17]. Pharmacodynamic studies in skin biopsies from patients taking $\geqslant 150 \mathrm{mg} \cdot$ day $^{-1}$ gefitinib revealed changes indicative of inhibition of the EGFR signalling pathway at every dose level evaluated $[13,19]$. Based on these results, the activity observed across the dose range and the more favourable tolerability at doses $\leqslant 600 \mathrm{mg} \cdot$ day $^{-1}$, two doses of gefitinib (250 and $500 \mathrm{mg} \cdot \mathrm{day}^{-1}$ ) were selected for phase II evaluation in patients with advanced pretreated NSCLC.

Towards the end of 2000, two uncontrolled, dose-randomised, double-blind, multicentre phase II studies were initiated to evaluate 250 and $500 \mathrm{mg} \cdot$ day $^{-1}$ doses of gefitinib in patients with locally advanced or metastatic NSCLC who had previously received platinum-based chemotherapy $[5,6]$ : the IRESSA Dose Evaluation in Advanced Lung Cancer (IDEAL) 1 $(n=210)$ and IDEAL $2(n=221)$ studies [5, 6]. Both studies showed that once daily oral treatment with gefitinib resulted in clinically meaningful anti-tumour activity, with objective response rates (ORRs) of $18 \%$ and $19 \%$ in IDEAL 1 and $12 \%$ and $9 \%$ in IDEAL 2 at 250 and $500 \mathrm{mg} \cdot \mathrm{day}^{-1}$, respectively. Disease-related symptom improvement rates were $40 \%$ and $37 \%$ among evaluable patients in IDEAL 1 and $43 \%$ and $35 \%$ in IDEAL 2, respectively. Median overall survival times were 7.6 and 8.0 months in IDEAL 1 and 7 and 6 months in IDEAL 2, respectively. The most common gefitinib-related adverse events were consistent with those observed in the phase I trials. Improved tolerability was noted at $250 \mathrm{mg} \cdot \mathrm{day}^{-1}$, with fewer patients requiring dose interruptions or dose reductions, withdrawing from treatment and experiencing grade $3 / 4$ events than at $500 \mathrm{mg} \cdot \mathrm{day}^{-1}(16 \%$ and $28 \%, 0 \%$ and $10 \%, 2 \%$ and $9 \%$, and $2 \%$ and $5 \%$ in IDEAL 1 , respectively). Thus, $250 \mathrm{mg} \cdot \mathrm{day}^{-1}$ was identified as the optimum biological dose for gefitinib and taken forward into the phase III clinical programme.

The concept of using optimal biological dose (rather than MTD dosing) for gefitinib is further supported by more recent clinical data, which have shown that, due to its pharmacokinetic properties, gefitinib concentrates more in tumour tissue (i.e. at the target) relative to plasma, reaching concentrations greater than 40-fold higher in breast tumour [20] and 60-fold higher in NSCLC tumour [21] than in coincident plasma samples.

Based on the phase II study results, the first marketing authorisation for gefitinib was granted in July 2002 in Japan for the treatment of inoperable or recurrent NSCLC.

\section{IDENTIFYING POTENTIAL PREDICTORS OF RESPONSE TO GEFITINIB}

The first clues as to which clinical subgroups of patients were most sensitive to gefitinib were provided by the phase II studies, in which higher ORRs were observed in patients with female sex and adenocarcinoma histology in IDEAL 1 and 2 [5, 6], and with Japanese ethnicity in IDEAL 1 [5]. A number of retrospective studies showed that never-smoking status was also associated with sensitivity to gefitinib [22-24]. However, as there were no control arms, it was not clear if similar results would be seen for any treatment or whether these were predictive factors specific to gefitinib.

Initial exploratory biomarker studies assessing EGFR protein expression using immunohistochemistry reported mixed results on the link between EGFR expression and outcome with gefitinib. While no relationship was found between EGFR protein expression and response for the IDEAL studies [25], other studies were inconclusive [26] or showed that EGFR protein expression was related to clinical outcome [27, 28]. Another potential biomarker investigated was EGFR gene copy number. An increase in EGFR gene copy number may occur as a result of polysomy (extra copies of chromosomes) or gene amplification (the presence of multiple copies of a gene) and can be investigated using fluorescence in situ hybridisation (FISH) [26]. Initial retrospective analyses showed an association between high EGFR gene copy number and outcome [27-29].

In 2004, sensitising mutations of EGFR were described in patients with advanced NSCLC [30-32]. In a retrospective analysis, $25(81 \%)$ of 31 patients who had experienced partial responses or marked clinical improvement on gefitinib or erlotinib (an EGFR-TKI) were found to have tumours with mutations in the EGFR tyrosine kinase domain [31]. Conversely, none of the 29 patients who did not respond to gefitinib or erlotinib was found to harbour EGFR mutations. These and subsequent studies showed that EGFR mutations were more prevalent in females, never-smokers, patients with adenocarcinoma histology and those of Asian ethnic origin [30-34], i.e. those groups in whom increased responsiveness to gefitinib had been observed.

In the early studies, EGFR mutational analyses of tumour samples were performed by the sequencing and analyses of PCR fragments in both sense and antisense directions for all exons of the EGFR tyrosine kinase domain. Two mutations in the EGFR tyrosine kinase domain (either multinucleotide inframe deletions of amino acids in exon 19 or point mutations resulting in a specific amino acid substitution at position 858 (L858R) in exon 21) were found to account for 49 (88\%) of 56 of the sensitising NSCLC-associated EGFR mutations [31]. As a small number of mutation types were shown to account for the vast majority of EGFR mutations, more sensitive targeted techniques have subsequently been developed that detect only the most common mutation types.

These initial results for EGFR biomarkers required further investigation in large scale, randomised, controlled, prospective studies.

\section{THE GEFITINIB MONOTHERAPY EXPERIENCE: FROM AN UNSELECTED PRETREATED POPULATION TO FIRST- LINE USE IN PATIENTS WITH EGFR MUTATION- POSITIVE NSCLC}

Initial phase III studies in unselected first-line NSCLC patients showed that gefitinib combined with platinum-based doublet chemotherapy gave no additional efficacy benefit over the 
TABLE 1 Summary of efficacy end-points for gefitinib from the IPASS, INTEREST and ISEL studies

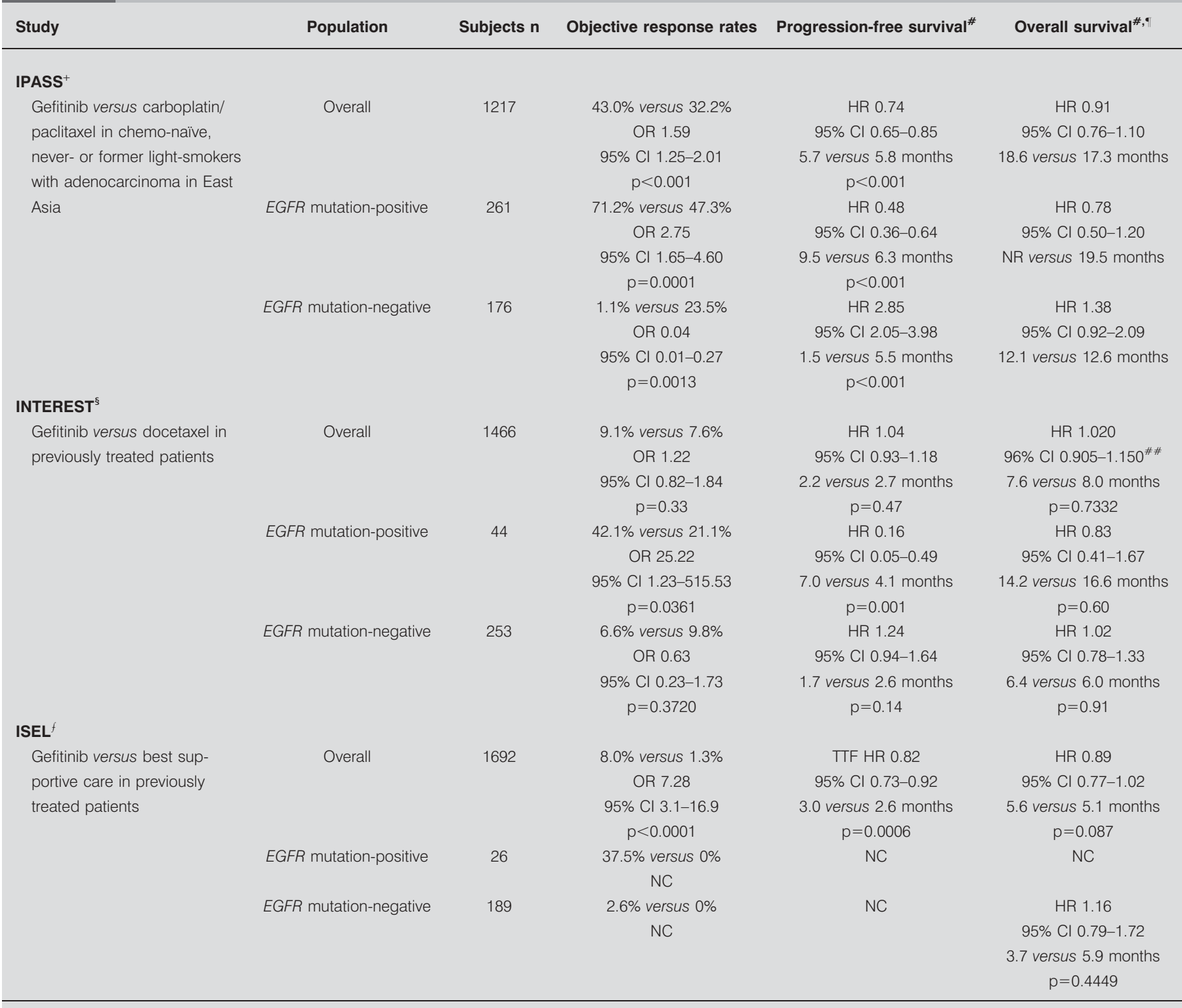

Odds ratios $>1$ favour gefitinib. Hazard ratios $<1$ favour gefitinib. IPASS: IRESSA Pan-Asia Study; INTEREST: IRESSA NSCLC Trial Evaluating Response and Survival versus Taxotere; ISEL: IRESSA Survival Evaluation in Lung cancer; EGFR: epidermal growth factor receptor; NR: not reached; NC: not calculated; TTF: time to treatment failure. ${ }^{\#}$ : median month values are presented; " : IPASS overall survival follow-up is ongoing; ${ }^{+}$: values presented for IPASS are for gefitinib versus carboplatin/paclitaxel; s. INTEREST values are for gefitinib versus docetaxel; ${ }^{f}$ : ISEL values are for gefitinib versus placebo; ${ }^{\# \#}$ : confidence interval entirely below non-inferiority margin of 1.154 . Data are taken from $[7,28,37,38,39]$ and previously unpublished studies.

chemotherapy regimen alone [35,36], and therefore the phase III programme progressed evaluating gefitinib as monotherapy.

\section{Gefitinib monotherapy versus placebo or docetaxel in pretreated patients}

Two phase III studies, ISEL and IRESSA NSCLC Trial Evaluating Response and Survival versus Taxotere (INTEREST), designed as phase IV commitments with the FDA to support the approval, evaluated the role of gefitinib monotherapy in pretreated patients. These studies were initiated in 2003 and early 2004, respectively, before the discovery of sensitising EGFR mutations, and recruited an unselected population. However, these two large studies provided the opportunity to evaluate the relationship between EGFR biomarkers and clinical outcome with gefitinib.

The placebo-controlled phase III ISEL study investigated the effect of gefitinib on survival for patients $(n=1,692)$ with locally advanced or metastatic NSCLC who had received one or two previous chemotherapy regimens and were refractory to or intolerant of their latest chemotherapy regimen [7]. Treatment with gefitinib was associated with a numerical 
improvement in survival in the overall unselected population, but this failed to reach statistical significance in the primary analysis (hazard ratio (HR) 0.89, 95\% CI 0.77-1.02; $\mathrm{p}=0.087$; table 1). The high proportion of chemotherapy refractory patients (90\%) in ISEL may account in part for this outcome in the overall population, as these patients represent a very difficult to treat population with a poor prognosis. However, pre-planned subgroup analyses showed statistically significant increases in survival with gefitinib compared with placebo for never-smokers ( $n=375$; HR 0.67, 95\% CI 0.49-0.92; $\mathrm{p}=0.012)$ and patients of Asian origin ( $\mathrm{n}=342$; HR $0.66,95 \%$ CI $0.48-0.91$; $\mathrm{p}=0.01$ ). The ORRs in the overall population were $8.0 \%$ versus $1.3 \%(\mathrm{p}<0.0001)$ for gefitinib and placebo, respectively, and preplanned subgroup analyses showed that the highest response rates with gefitinib were among never-smokers (18.1\%), females $(14.7 \%)$, patients of Asian origin (12.4\%) and patients with adenocarcinomas $(11.9 \%)$; the characteristics that are now known to be typically associated with increased incidence of EGFR mutation. Gefitinib was well tolerated, with the most common adverse events being rash (37\% versus $10 \%)$ and diarrhoea ( $27 \%$ versus $9 \%$ ); mostly CTC grade 1 or 2 in severity.

Based on the ISEL results, in June 2005, the FDA limited the use of gefitinib to patients who have previously taken gefitinib and are benefiting or have benefited from gefitinib, and AstraZeneca withdrew its marketing authorisation application under review in Europe. Given the more favourable results observed for patients of Asian ethnicity, the drug remained available in many Asian countries.

A panel of EGFR-related biomarkers was subsequently investigated in 460 tumour samples from patients in the ISEL study [28]. High EGFR gene copy number measured by FISH was found to be a predictor of a survival benefit with gefitinib compared with placebo (HR 0.61 and 1.16 for high and low copy number, respectively; interaction test (comparison of high versus low copy number HR) $p=0.045$ ). An association was also observed between EGFR protein expression and survival (HR 0.77 and 1.57 for positive and negative expression, respectively; interaction test $\mathrm{p}=0.049)$. There were insufficient EGFR mutation-positive samples for survival analysis by EGFR mutation status, although gefitinib-treated patients with mutations had higher ORRs than those without (table 1). Of all the clinical and biomarker subgroups assessed, gefitinib ORR was highest in the EGFR mutation-positive subgroup [7, 28].

In the INTEREST study, first reported in late 2007 [40], patients with locally advanced or metastatic NSCLC that had progressed or recurred after one or two previous platinum-based chemotherapy regimens were randomised to treatment with either gefitinib $(n=733)$ or docetaxel $(n=733)$ [37]. Non-inferior survival of gefitinib compared with docetaxel was demonstrated in the overall unselected population (HR 1.020, 96\% CI 0.905-1.150; predefined non-inferiority margin 1.154) (table 1). Progression-free survival (PFS) and ORR were similar in both treatment groups (table 1). Significantly more patients had improvements in quality of life (QoL) with gefitinib compared with docetaxel as assessed by Functional Assessment of Cancer Therapy-Lung (FACT-L) total score (OR 1.99, 95\% CI 1.42-2.79; $\mathrm{p}<0.0001)$ and the FACT-L Trial Outcome Index (TOI) (OR 1.82, 95\% CI 1.23-2.69; $\mathrm{p}=0.0026)$, and there was no significant difference between the two arms in the proportions of patients who had improvements in lung cancer symptoms $(p=0.13)$. Some clinical factors (never-smoking, Asian origin, female sex and adenocarcinoma histology) were associated with long survival, although similar effects were seen with both gefitinib and docetaxel and so no significant differences between treatments were observed (fig. 2). However, there was more heterogeneity in the treatment differences for the secondary end-points of PFS and ORR (fig. 2). Gefitinib had a more favourable tolerability profile than docetaxel. The most common adverse events were rash/acne (49\% versus $10 \%$ ) and diarrhoea ( $35 \%$ versus $25 \%$ ) in the gefitinib group, and neutropenia $(5 \%$ versus $74 \%$ ), asthenic disorders (25\% versus $47 \%$ ) and alopecia ( $3 \%$ versus $36 \%$ ) in the docetaxel group. Gefitinib was associated with lower rates of grade 3 or 4 adverse events ( $9 \%$ versus $41 \%$ ), particularly grade 3 or 4 neutropenia ( $2 \%$ versus $58 \%$ ). Other studies have consistently shown that the efficacy of gefitinib is similar to that of docetaxel in unselected patient populations in the pretreated setting, but with an improved tolerability and QoL profile [41-43].

The biomarker findings from ISEL prompted the introduction of a co-primary analysis of superior overall survival in patients with high EGFR gene copy number in the INTEREST study, via protocol amendment in 2006. Superior overall survival of gefitinib versus docetaxel in patients with high EGFR gene copy number was not proven (HR 1.09, 95\% CI 0.78-1.51; $\mathrm{p}=0.62$ ). One possible explanation for the difference in biomarker results between ISEL and INTEREST is that gefitinib and docetaxel have similar activity in patients with high or low EGFR gene copy number and that high EGFR gene copy number is predictive of a greater survival benefit over placebo for both treatments [38]. Another is that crossover to the alternative therapy at disease progression made it more difficult to detect an overall survival difference in INTEREST as many patients received both treatments. In contrast to overall survival, PFS and ORR advantages for gefitinib tended to be larger in patients with high EGFR gene copy number compared with those with low copy number [38]. There was no evidence of a difference in overall survival, PFS or ORR between treatments according to EGFR protein expression status. Of 297 patients with samples evaluable for EGFR mutations, $44(15 \%)$ were EGFR mutation positive. Among patients with EGFR mutation-positive tumours, PFS and ORR were higher for gefitinib compared with docetaxel (table 1 and fig. 2). However, overall survival was similarly long with both gefitinib and docetaxel in patients with EGFR mutationpositive tumours and, hence, there was no statistically significant difference in overall survival between treatments in the small EGFR mutation-positive subgroup (HR 0.83, 95\% CI $0.41-1.67 ; \mathrm{p}=0.60$ ) (table 1). A prospective trial with a higher number of patients with EGFR mutation-positive status was needed to make any definitive conclusions regarding EGFR mutation status and outcome.

\section{Gefitinib versus doublet chemotherapy as first-line treatment in clinically selected patients}

Although EGFR mutations had been identified at the time of designing the IPASS trial in 2005, the importance of these and other biomarkers relative to clinical characteristics was not clear at that point. Therefore, patients were selected for IPASS on the basis of clinical characteristics known to be associated 


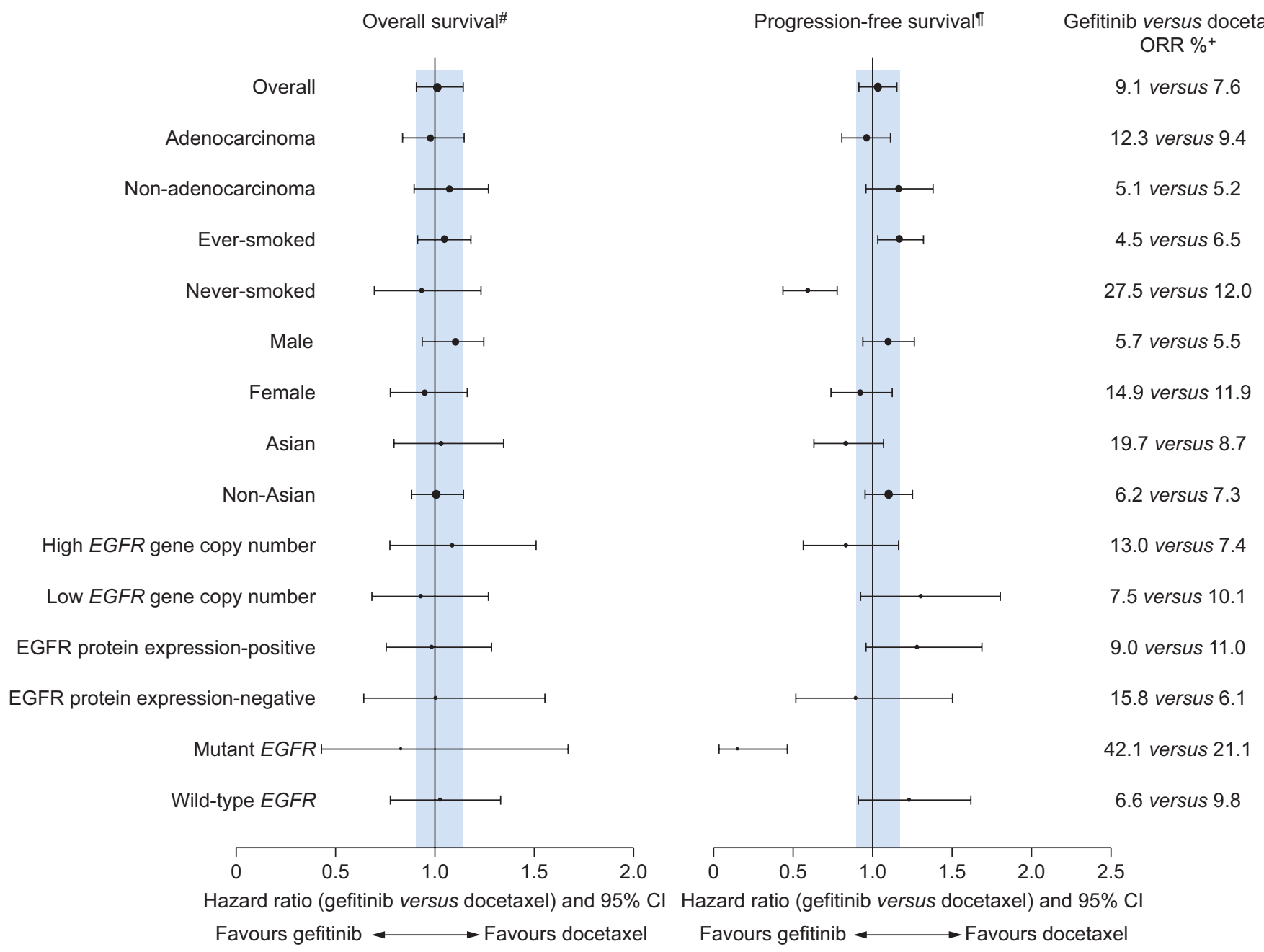

FIGURE 2. Forest plot of (left) overall survival and (right) progression-free survival for gefitinib versus docetaxel in the INTEREST (IRESSA NSCLC Trial Evaluating Response and Survival versus Taxotere) study (pretreated setting) by clinical characteristics and epidermal growth factor receptor (EGFR) biomarkers [37, 38]. ORR: objective response rate. ${ }^{*}$ : unadjusted analysis - per-protocol population for clinical factors and intent-to-treat population for biomarker factors; " response (EFR) population; ${ }^{+}$: EFR population.

with increased response to gefitinib. Never-smokers or former light-smokers in East Asia who had adenocarcinoma of the lung were randomised, between March 2006 and October 2007, to receive either gefitinib $(n=609)$ or carboplatin/paclitaxel $(n=608)$ as first-line treatment [39]. The study met its primary objective of demonstrating non-inferiority and additionally showed the superiority of gefitinib compared with carboplatin/paclitaxel for PFS in the overall clinically selected population (HR 0.74, 95\% CI 0.65-0.85; p<0.001) (table 1). The effect was not constant over time, with the probability of being progression-free in favour of carboplatin/paclitaxel in the first 6 months, and in favour of gefitinib in the following 16 months, likely driven by the differing effects in subgroups (see later). The ORR was significantly higher with gefitinib compared with carboplatin/paclitaxel (table 1) and significantly more patients had improvements in QoL with gefitinib compared with carboplatin/paclitaxel as assessed by FACT-L total score (OR 1.34, 95\% CI 1.06-1.69; $\mathrm{p}=0.01$ ) and the FACT-L TOI (OR 1.78, 95\% CI 1.40-2.26; $\mathrm{p}<0.001$ ). Similar proportions of patients had improvements in symptoms as assessed by
FACT-L Lung Cancer Subscale (LCS) score (OR 1.13, 95\% CI $0.90-1.42 ; \mathrm{p}=0.30)$. As in previous studies, gefitinib had a more favourable tolerability profile than chemotherapy, with a lower rate of grade 3 or 4 adverse events (29\% versus $61 \%$ ). The most common adverse events in the gefitinib group were rash or acne $(66 \%$ versus $22 \%)$ and diarrhoea ( $47 \%$ versus $22 \%$ ), whereas in the carboplatin/paclitaxel group, the most common adverse events were neurotoxic effects ( $11 \%$ versus $70 \%$ ), neutropenia ( $4 \%$ versus $67 \%$ ) and alopecia ( $11 \%$ versus $58 \%$ ).

Analysis of efficacy according to baseline biomarker status was a preplanned objective of IPASS. Samples were tested for the presence of EGFR mutations using the amplification refractory mutation system (the DxS EGFR29 mutation-detection kit; Qiagen, formerly DxS, Manchester, UK) (437 were evaluable for EGFR mutation status). The presence of EGFR mutation was a very strong predictor of improved PFS and ORR with gefitinib compared with carboplatin/paclitaxel. Among the subgroup of 261 patients who had EGFR mutation-positive tumours, PFS was significantly longer with gefitinib compared 


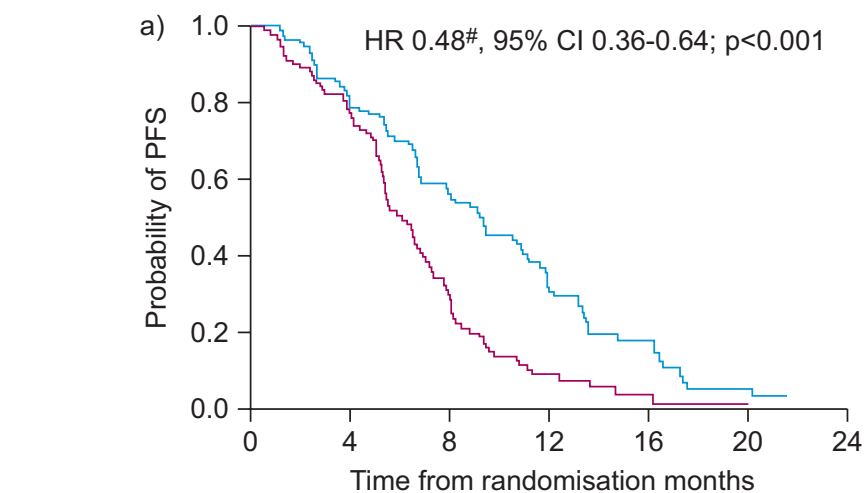

Number at risk:

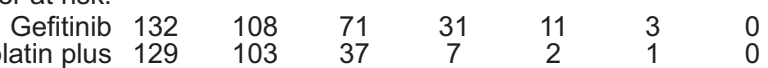
paclitaxel

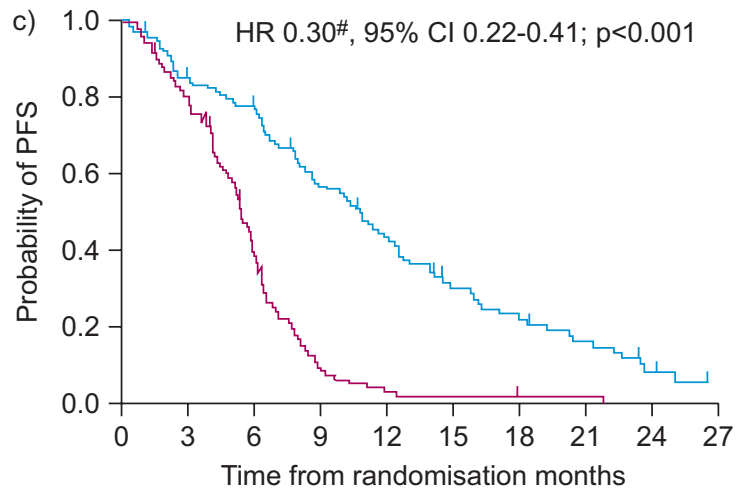

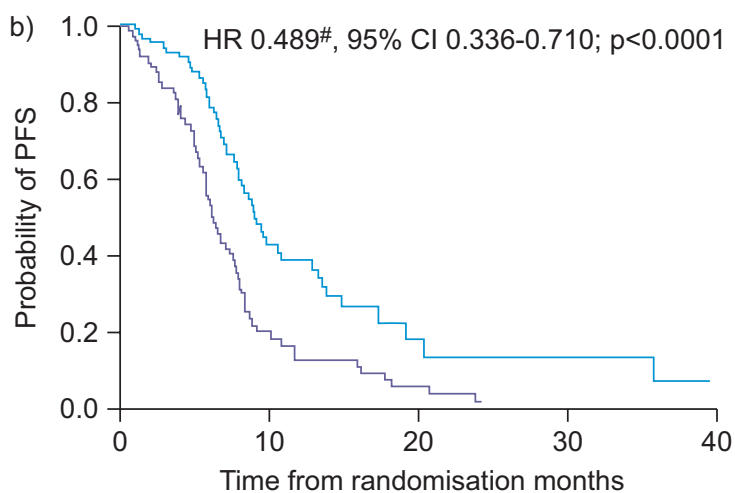

Number at risk:

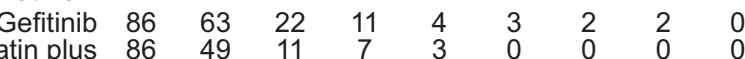
docetaxel

FIGURE 3. Kaplan-Meier curve for progression-free survival for gefitinib versus doublet chemotherapy in three phase III trials in first-line nonsmall cell lung cancer harbouring an activating epidermal growth factor receptor (EGFR) mutation: a) patients with EGFR mutation-positive status in the IRESSA Pan-Asia Study (IPASS; first-line setting) (—_ gefitinib; —_ carboplatin plus paclitaxel) (reproduced from [39] with permission from the publisher; @ 2009 Massachusetts Medical Society. All rights reserved), b) the overall population (all EGFR mutation-positive) in the WJTOG3405 study (first-line setting) (—_: gefitinib; ——: cisplatin plus docetaxel) (reproduced from [44] with permission from the publisher; @ 2010, with permission from Elsevier) and c) the overall population (all EGFR mutation-positive) in the NEJ002 study (first-line setting) (— : gefitinib $(n=114)$; — : carboplatin plus paclitaxel $(n=110)$ ) (reproduced from [45] with permission from the publisher; $\odot$ 2009 Massachusetts Medical

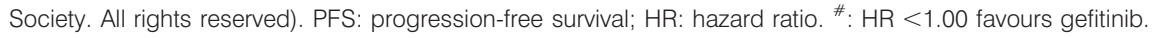

with carboplatin/paclitaxel (HR $0.48,95 \%$ CI $0.36-0.64$; $\mathrm{p}<0.001$ ) (table 1 and fig. 3a). Conversely, in the subgroup of 176 patients with EGFR mutation-negative tumours, PFS was significantly longer with carboplatin/paclitaxel compared with gefitinib (HR 2.85, 95\% CI 2.05-3.98; p<0.001) (table 1). The treatment by mutation status interaction test $p$-value showed $p<0.001$. The benefit seen with gefitinib in the overall study population was driven primarily by the subgroup of patients with EGFR mutations, with ORRs of $71.2 \%$ versus $1.1 \%$ in mutation-positive versus mutation-negative subgroups (table 1). The ORRs with carboplatin/paclitaxel were $47.3 \%$ versus $23.5 \%$, respectively (table 1 ).

Unplanned analysis of the relatively small amount of early overall survival data available at the time of the primary PFS analysis by mutation status showed a HR numerically in favour of gefitinib in the EGFR mutation-positive subgroup (HR 0.78, 95\% CI 0.50-1.20; based on 81 events) (table 1) and numerically in favour of carboplatin/paclitaxel (HR 1.38, 95\% CI 0.92-2.09; based on 94 events) (table 1) in the mutationnegative subgroup. This preliminary analysis was based on a relatively small number of events. Further follow-up for mature overall survival data is ongoing. However, even when the data are mature, analysis of the overall survival data is likely to be confounded by crossover to the comparator treatment; for this reason PFS was chosen as the primary outcome of this study. Additionally, as shown by BROGLIO and BERRY [46], even when overall survival is improved it can be difficult to demonstrate statistical significance if survival postprogression is long, such as longer than 12 months; this was the case for the IPASS mutation-positive subgroup, in which median PFS on the chemotherapy arm was 6.3 months but median overall survival was 19.5 months.

Significantly more patients in the EGFR mutation-positive subgroup had improvements in QoL with gefitinib compared with carboplatin/paclitaxel as assessed by FACT-L total score (OR 3.01, 95\% CI 1.79-5.07; $\mathrm{p}<0.0001)$ and FACT-L TOI (OR 3.96, 95\% CI 2.33-6.71; $\mathrm{p}<0.0001$ ), and in symptoms as assessed by FACT-L LCS score (OR 2.70, 95\% CI 1.58 $4.62 ; \mathrm{p}=0.0002)$. Conversely, in the mutation-negative subgroup, significantly more patients had improvements in QoL and symptoms with carboplatin/paclitaxel compared with gefitinib. 
Analysis of PFS by EGFR gene copy number (measured by FISH) produced similar but less marked trends to those observed in the EGFR mutation analysis [47]. The treatment by gene copy number interaction test showed $p=0.0437$. PFS was significantly longer with gefitinib than with carboplatin/ paclitaxel in the subgroup of 249 patients with high EGFR gene copy number (HR 0.66, 95\% CI 0.50-0.88; p=0.0050) and numerically longer with carboplatin/paclitaxel than with gefitinib in the subgroup of 157 patients with low EGFR gene copy number (HR 1.24, 95\% CI 0.87-1.76; $\mathrm{p}=0.2368$ ). However, there was a high degree of overlap between EGFR mutationpositivity and high EGFR gene copy number: of 245 patients with high EGFR gene copy number whose EGFR mutation status was also known, 190 (78\%) were also EGFR mutation positive. This suggests that the improved outcome in high EGFR gene copy number patients is being driven by the EGFR mutation-positive overlap. There was no relationship evident between EGFR protein expression status and PFS outcome.

In summary, patients selected by clinical characteristics for first-line treatment in IPASS had prolonged PFS and increased ORR with gefitinib. The finding that PFS treatment effect changed over time (favouring carboplatin/paclitaxel for the first 6 months and gefitinib thereafter) is likely due to the mixed population with regard to EGFR mutation status, the initial advantage for carboplatin/paclitaxel being attributed to the benefit of chemotherapy over gefitinib in the EGFR mutation-negative subgroup and the subsequent advantage for gefitinib attributed to the prolonged PFS in the EGFR mutationpositive subgroup with gefitinib. The presence of EGFR mutation was the strongest and most reliable predictor of improved PFS and ORR with gefitinib compared with carboplatin/paclitaxel as first-line therapy of advanced NSCLC.

The results in the EGFR mutation-positive subgroup from the IPASS trial are now supported by those of two other phase III randomised controlled trials conducted in Japan of gefitinib versus doublet chemotherapy as first-line treatment of

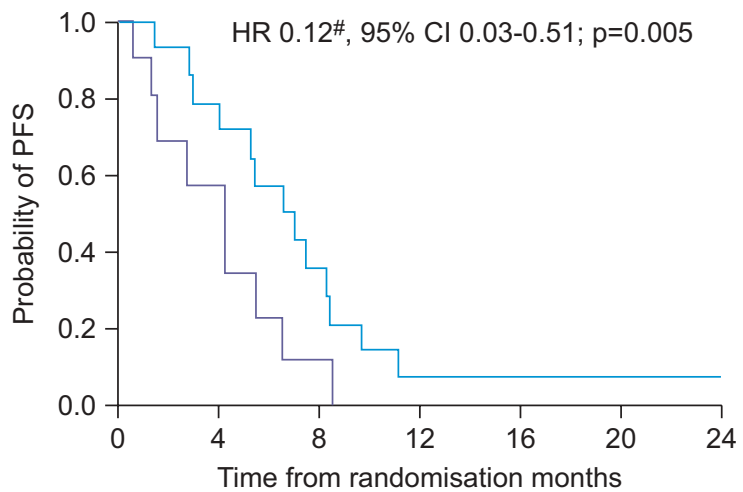

Number at risk:

$\begin{array}{cccccccc}\text { Gefitinib } & 14 & 11 & 5 & 1 & 1 & 1 & 1 \\ \text { Docetaxel } & 10 & 5 & 1 & 0 & 0 & 0 & 0\end{array}$

FIGURE 4. Kaplan-Meier curve for progression-free survival for non-Asian patients with epidermal growth factor receptor (EGFR) mutation-positive status in the INTEREST (IRESSA NSCLC Trial Evaluating Response and Survival versus Taxotere) study (pretreated setting). ——: gefitinib; — $\longrightarrow$ : docetaxel. PFS: progression-free survival; HR: hazard ratio. ${ }^{\#}$ : HR $<1.00$ favours gefitinib. Reproduced from [53] with permission from the publisher. advanced NSCLC harbouring EGFR sensitising mutations $[44,45]$. In these two studies, gefitinib significantly prolonged PFS compared with doublet chemotherapy $(n=172$; HR 0.489, 95\% CI $0.336-0.710 ; \quad \mathrm{p}<0.0001$ versus cisplatin/docetaxel (fig. 3b); and $\mathrm{n}=224$; HR $0.30,95 \%$ CI $0.22-0.41 ; \mathrm{p}<0.001$ versus carboplatin/paclitaxel (fig. 3c)) [44, 45].

Evidence suggests that patients with sensitising EGFR mutations have high response rates to gefitinib irrespective of ethnicity [48], although the proportion of patients with EGFR mutations is lower for non-Asian $(\sim 10-15 \%)[49,50]$ compared with Asian patients ( 30-40\%) [51, 52]. A post hoc subgroup analysis of the INTEREST study (pretreated patients) showed that PFS was significantly longer with gefitinib than docetaxel in non-Asian patients with EGFR mutation-positive disease, although the patient numbers were low (fig. 4).

The IPASS result presents a paradigm shift in the treatment of lung cancer: a first-line oral treatment option for patients with EGFR mutation-positive tumours that is more effective than doublet chemotherapy, the standard of care as first-line treatment for advanced NSCLC since the 1980s. By targeting the EGFR, a receptor preferentially expressed by tumour cells, gefitinib is associated with predictable and more favourable tolerability compared with traditional chemotherapy that interferes with all rapidly dividing cells. In June 2009, the EMA granted marketing authorisation for gefitinib for adults with locally advanced or metastatic NSCLC with activating mutations of EGFR-tyrosine kinase, based on a submission package including the IPASS and INTEREST studies.

\section{LESSONS LEARNED}

The sooner a predictive biomarker for the effect of a new treatment is identified, the more focused and efficient the clinical development programme can become. One of the major challenges in the development of gefitinib was that scientific understanding of the drug's target biology, and potential biomarkers of outcome, advanced in conjunction with its clinical development. Consequently, the identification of clinical characteristics and biomarkers of response to gefitinib, and the refinement of these, occurred simultaneously to a large degree. Trials in unselected NSCLC populations first revealed clinical characteristics and then biomarkers associated with high response rates, ultimately leading to prospective studies of gefitinib in patients with EGFR mutation-positive disease. We now know that it is EGFR mutation rather than protein expression or gene copy number that is linked to the dependency on the EGFR pathway and, therefore, the mechanism of action of gefitinib. Sensitising mutations of the EGFR increase the activation and duration of receptor signalling after ligand binding compared with the wild-type receptor [32]. The mutated EGFR exhibits preferential signalling down the AKT pathway and this gives the cell a survival advantage that must be maintained. Over time, the tumour becomes heavily dependent on this target and oncogenic addiction occurs [54].

It is hoped that, in the future, technical advances will result in preclinical models that are better able to identify optimal biomarkers for response to new targeted anti-cancer therapies, enabling more efficient clinical development. In the absence of a targeted biomarker in early clinical development, a surrogate for the biomarker may help to identify those patients most 


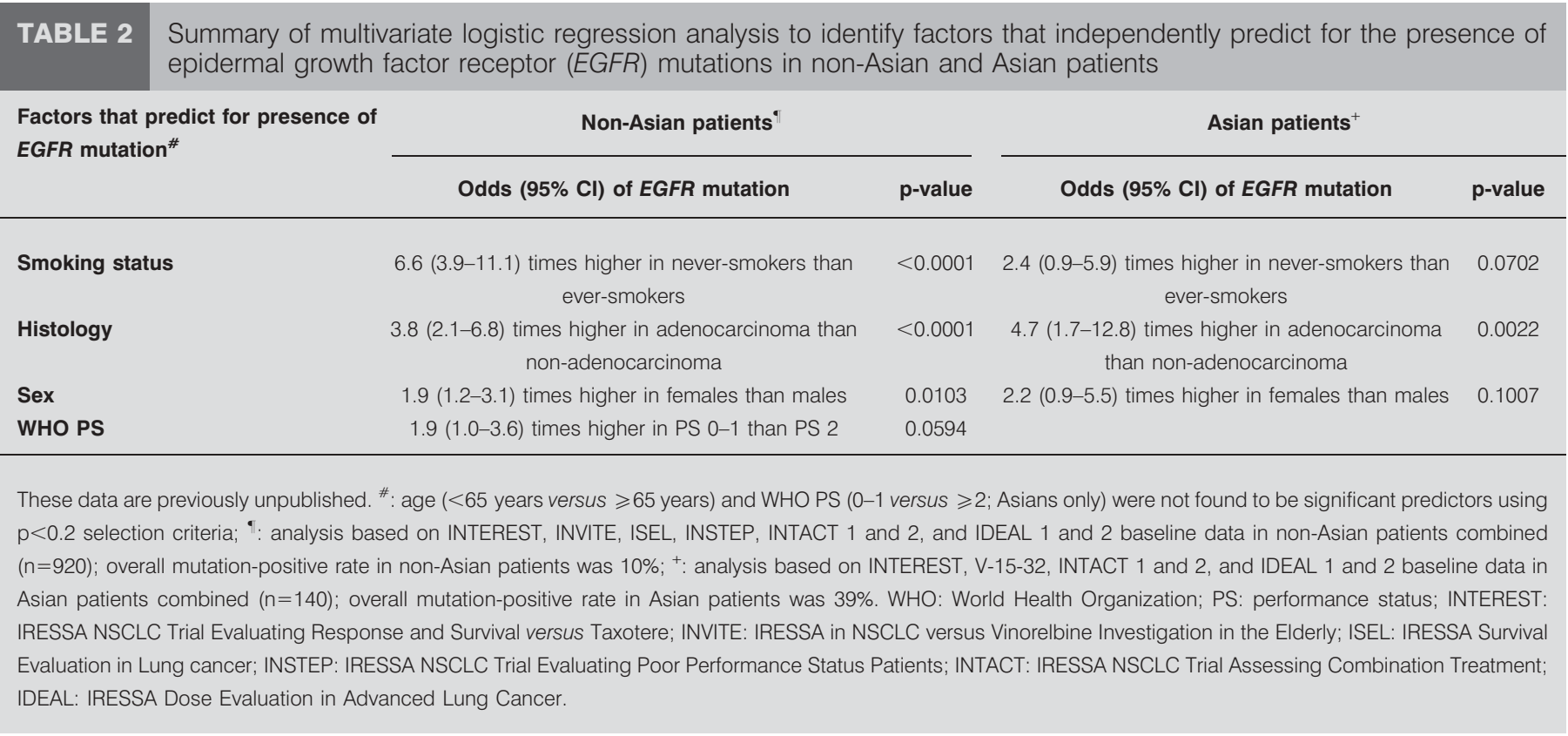

likely to respond to treatment. Current evidence suggests that the early identified potential markers of outcome to gefitinib, including clinical characteristics and EGFR gene copy number, were probably surrogates for EGFR mutation status [47]. Indeed, a multivariate logistic regression analysis (to identify factors that independently predicted for the presence of EGFR mutations in a total of 1,060 patients included in clinical trials of gefitinib) confirmed that the clinical characteristics of neversmoker, adenocarcinoma histology and female sex are independent predictors of positive EGFR mutation status (table 2).

In the IPASS trial, patients were selected on clinical characteristics (never- or former light-smoker, adenocarcinoma histology and living in Asia), and $60 \%$ of those patients with evaluable samples were found to be EGFR mutation positive [39]. While the study showed a significant PFS benefit with gefitinib compared with carboplatin/paclitaxel overall and in those patients who harboured an EGFR mutation, patients without the mutation did better on chemotherapy. Therefore, clinical characteristics cannot be considered to be appropriate surrogates for EGFR mutation status when making treatment decisions regarding use of gefitinib versus doublet chemotherapy in the first-line setting. Nevertheless, clinical surrogates may be useful in other settings, for other drugs and biomarkers.

If biomarkers could potentially affect treatment outcome, then ideally, high quality tissue samples should be collected from all patients in the study. This maximises the ability to detect predictive biomarkers if they exist. However, the gefitinib development programme highlights the practical challenges in conducting biomarker research. These are mainly related to collection of adequate numbers of tissue samples that are of sufficiently high quality and quantity, which is particularly problematic in the case of lung cancer, where the tumour is relatively inaccessible, some patients have inoperable disease and where routine clinical practice does not necessarily generate the samples required for biomarker analysis. Other challenges include the informed consent process for biomarker evaluation, problems with incomplete pathology tracking consent and recovery of sufficient DNA from the samples for EGFR mutation analysis. In the case of multinational phase III trials, sample quality can be variable and the process of obtaining informed consent presents different challenges in different countries. For example, in the IPASS trial, despite $1,038(85 \%)$ of the 1,217 trial participants providing consent for biomarker analyses, tissue samples were provided for only 683 of these, of which 437 were evaluable for EGFR mutations and 261 were found to have EGFR mutation-positive tumours [39]. In non-Asian populations, the difficulties are further compounded by the lower prevalence of EGFR mutations $(\sim 10$ $15 \%)[49,50]$. In the ISEL and INTEREST studies, insufficient EGFR mutation-positive tissue samples were identified for the definitive determination of whether EGFR mutation status was a predictive factor for the efficacy of gefitinib. If biomarkers might be important factors determining response, then maximising the number of high-quality tissue samples should be considered high priority in the design of the clinical programme, and studies should be sufficiently powered to detect clinically important differences in biomarker subgroups. Protocols and consents also need to allow for future developments in tests and technologies, as the science can evolve rapidly while trials are ongoing.

Another important requisite is a readily available and accurate diagnostic test for the biomarker that determines response, and the use of a consistent definition of positivity (cut-off). Three methods of measuring EGFR and determining outcome were assessed during the development of gefitinib: EGFR protein expression, EGFR gene copy number and EGFR mutation. It took some time to demonstrate that EGFR mutation was the strongest predictive factor in identifying patients that would benefit from gefitinib. A number of techniques for detecting EGFR mutation have been evaluated with gefitinib and new less invasive approaches for the patient, such as detection in cytology and serum samples, continue to be assessed. 
It is possible that randomised controlled phase II trials would have enabled more rapid identification of the subgroups on which to focus gefitinib development, as they are more informative than uncontrolled trials. A predictive factor can only be identified from a controlled trial, and not from a single-arm study.

Finally, early engagement with regulatory agencies is important to ensure that the planned development programme will meet their requirements in this rapidly evolving area. Both the regulatory agencies and pharmaceutical companies are learning about the development of biomarker-targeted agents together and early discussions may facilitate opportunities for collaboration.

\section{SUMMARY}

The development of a new molecularly targeted agent represents a significant challenge, as the knowledge about the target is often limited at the time of designing the clinical programme. In fact, the drug under development may serve as a tool to further explore and improve understanding of the target biology. Gefitinib is now approved in Europe for the treatment of adults with locally advanced or metastatic NSCLC with activating mutations of EGFR tyrosine kinase. In various other countries, particularly those in Asia, gefitinib is indicated in an unselected pretreated NSCLC population and in some countries, the addition of a first-line indication in patients with activating mutations of EGFR tyrosine kinase has recently been granted.

For the first time, an oral treatment offers superior efficacy, in terms of PFS and ORR, and better tolerability and QoL compared with doublet chemotherapy (carboplatin/paclitaxel) as first-line treatment for patients with advanced NSCLC harbouring EGFR mutations. Physicians now have a test that will give a good indication that a treatment for lung cancer will work, which is in contrast to the usual position of administering chemotherapy and hoping that their patient will respond well to the chosen treatment.

However, it took several years and many large studies before the target patient population for gefitinib treatment in advanced NSCLC became clear. There are several useful lessons for future biomarker-targeted products from the gefitinib experience: understanding the science is fundamental in determining which biomarker the tumour is truly dependent on and how best to measure that biomarker; the number of tissue samples collected in trials needs to be maximised so that the significance of the biomarker can be fully evaluated; and the diagnostic test needs to be available together with the drug. Pharmaceutical companies and regulatory agencies will need to engage early on and work collaboratively to ensure that efficacious and safe personalised medicines are available to patients in a timely manner, without the requirement for excessively long and complex development programmes.

\section{STATEMENT OF INTEREST}

A.A. Armour and C.L. Watkins are employed by and own shares in AstraZeneca, UK.

\section{ACKNOWLEDGEMENTS}

We thank A. Smith from Complete Medical Communications, Macclesfield, UK who provided medical writing support funded by AstraZeneca.

\section{REFERENCES}

1 Ferlay J, Bray F, Pisani P, et al. GLOBOCAN 2002: Cancer incidence, mortality and prevalence worldwide. IARC CancerBase No. 5. version 2.0. Lyon, IARC Press, 2004.

2 Jemal A, Siegel R, Ward E, et al. Cancer statistics, 2009. CA Cancer J Clin 2009; 59: 225-249.

3 Non-small Cell Lung Cancer Collaborative Group. Chemotherapy in non-small cell lung cancer: a meta-analysis using updated data on individual patients from 52 randomised clinical trials. BMJ 1995; 311: 899-909.

4 NSCLC Meta-Analyses Collaborative Group. Chemotherapy in addition to supportive care improves survival in advanced nonsmall-cell lung cancer: a systematic review and meta-analysis of individual patient data from 16 randomized controlled trials. J Clin Oncol 2008; 26: 4617-4625.

5 Fukuoka M, Yano S, Giaccone G, et al. Multi-institutional randomized phase II trial of gefitinib for previously treated patients with advanced non-small-cell lung cancer. J Clin Oncol 2003; 21: 2237-2246.

6 Kris MG, Natale RB, Herbst RS, et al. Efficacy of gefitinib, an inhibitor of the epidermal growth factor receptor tyrosine kinase, in symptomatic patients with non-small cell lung cancer. A randomized trial. JAMA 2003; 290: 2149-2158.

7 Thatcher N, Chang A, Parikh P, et al. Gefitinib plus best supportive care in previously treated patients with refractory advanced non-small-cell lung cancer: results from a randomised, placebo-controlled, multicentre study (Iressa Survival Evaluation in Lung Cancer). Lancet 2005; 366: 1527-1537.

8 Salomon DS, Brandt R, Ciardiello F, et al. Epidermal growth factorrelated peptides and their receptors in human malignancies. Crit Rev Oncol Hematol 1995; 19: 183-232.

9 Woodburn JR. The epidermal growth factor receptor and its inhibition in cancer therapy. Pharmacol Ther 1999; 82: 241-250.

10 Ciardiello F, Tortora G. EGFR antagonists in cancer treatment. N Engl J Med 2008; 358: 1160-1174.

11 Baselga J. New technologies in epidermal growth factor receptortargeted cancer therapy. Signal 2000; 1: 12-21.

12 Wakeling AE, Guy SP, Woodburn JR, et al. ZD1839 (Iressa): an orally active inhibitor of epidermal growth factor signaling with potential for cancer therapy. Cancer Res 2002; 62: 5749-5754.

13 Baselga J, Rischin D, Ranson M, et al. Phase I safety, pharmacokinetic, and pharmacodynamic trial of ZD1839, a selective oral epidermal growth factor receptor tyrosine kinase inhibitor, in patients with five selected solid tumor types. J Clin Oncol 2002; 20: 4292-4302.

14 Herbst RS, Maddox AM, Rothenberg ML, et al. Selective oral epidermal growth factor receptor tyrosine kinase inhibitor ZD1839 is generally well-tolerated and has activity in non-small-cell lung cancer and other solid tumors: results of a phase I trial. J Clin Oncol 2002; 20: 3815-3825.

15 Nakagawa K, Tamura T, Negoro S, et al. Phase I pharmacokinetic trial of the selective oral epidermal growth factor receptor tyrosine kinase inhibitor gefitinib ("Iressa", ZD1839) in Japanese patients with solid malignant tumours. Ann Oncol 2003; 14: 922-930.

16 Ranson M, Hammond LA, Ferry D, et al. ZD1839, a selective oral epidermal growth factor receptor-tyrosine kinase inhibitor, is well tolerated and active in patients with solid, malignant tumors: results of a phase I trial. J Clin Oncol 2002; 20: 2240-2250.

17 Wolf M, Swaisland H, Averbuch S. Development of the novel biologically targeted anticancer agent gefitinib: determining the optimum dose for clinical efficacy. Clin Cancer Res 2004; 10: 4607-4613.

18 Rukazenkov Y, Speake G, Marshall G, et al. Epidermal growth factor receptor tyrosine kinase inhibitors: similar but different? Anticancer Drugs 2009; 20: 856-866.

19 Albanell J, Rojo F, Averbuch S, et al. Pharmacodynamic studies of the epidermal growth factor receptor inhibitor ZD1839 in skin 
from cancer patients: histopathologic and molecular consequences of receptor inhibition. J Clin Oncol 2002; 20: 110-124.

20 McKillop D, Partridge EA, Kemp JV, et al. Tumor penetration of gefitinib (Iressa), an epidermal growth factor receptor tyrosine kinase inhibitor. Mol Cancer Ther 2005; 4: 641-649.

21 Haura E, Sommers E, Becker D, et al. Pilot phase II study of preoperative gefitinib in early stage non-small cell lung cancer with assessment of intratumour gefitinib levels and tumour target modulation. J Clin Oncol 2007; 25: Suppl. 18, 7603.

22 Kaneda H, Tamura K, Kurata T, et al. Retrospective analysis of the predictive factors associated with the response and survival benefit of gefitinib in patients with advanced non-small-cell lung cancer. Lung Cancer 2004; 46: 247-254.

23 Miller VA, Kris MG, Shah N, et al. Bronchioloalveolar pathologic subtype and smoking history predict sensitivity to gefitinib in advanced non-small-cell lung cancer. J Clin Oncol 2004; 22: 1103-1109.

24 Takano $\mathrm{T}$, Ohe $\mathrm{Y}$, Kusumoto $\mathrm{M}$, et al. Risk factors for interstitial lung disease and predictive factors for tumor response in patients with advanced non-small cell lung cancer treated with gefitinib. Lung Cancer 2004; 45: 93-104.

25 Bailey LR, Kris M, Wolf M, et al. Tumor EGFR membrane staining is not clinically relevant for predicting response in patients receiving gefitinib ("Iressa", ZD1839) monotherapy for pretreated advanced non-small-cell lung cancer: IDEAL 1 and 2. Proc Am Assoc Cancer Res 2003; 44: LB-170.

26 Hirsch FR, Varella-Garcia M, Bunn PA Jr, et al. Epidermal growth factor receptor in non-small-cell lung carcinomas: correlation between gene copy number and protein expression and impact on prognosis. J Clin Oncol 2003; 21: 3798-3807.

27 Cappuzzo F, Hirsch FR, Rossi E, et al. Epidermal growth factor receptor gene and protein and gefitinib sensitivity in non-smallcell lung cancer. J Natl Cancer Inst 2005; 97: 643-655.

28 Hirsch FR, Varella-Garcia M, Bunn Jr PA, et al. Molecular predictors of outcome with gefitinib in a phase III placebocontrolled study in advanced non-small-cell lung cancer. J Clin Oncol 2006; 24: 5034-5042.

29 Hirsch FR, Varella-Garcia M, McCoy J, et al. Increased epidermal growth factor receptor gene copy number detected by fluorescence in situ hybridization associates with increased sensitivity to gefitinib in patients with bronchioloalveolar carcinoma subtypes: a Southwest Oncology Group study. J Clin Oncol 2005; 23: 6838-6845.

30 Paez JG, Jänne PA, Lee JC, et al. EGFR mutations in lung cancer: correlation with clinical response to gefitinib therapy. Science 2004; 304: 1497-1500.

31 Pao W, Miller V, Zakowski M, et al. EGF receptor gene mutations are common in lung cancers from "never smokers" and are associated with sensitivity of tumors to gefitinib and erlotinib. Proc Natl Acad Sci USA 2004; 101: 13306-13311.

32 Lynch TJ, Bell DW, Sordella R, et al. Activating mutations in the epidermal growth factor receptor underlying responsiveness of non-small-cell lung cancer to gefitinib. N Engl J Med 2004; 350: 2129-2139.

33 Kosaka T, Yatabe Y, Endoh H, et al. Mutations of the epidermal growth factor receptor gene in lung cancer: biological and clinical implications. Cancer Res 2004; 64: 8919-8923.

34 Shigematsu $\mathrm{H}$, Lin L, Takahashi $\mathrm{T}$, et al. Clinical and biological features associated with epidermal growth factor receptor gene mutations in lung cancers. J Natl Cancer Inst 2005; 97: 339-346.

35 Giaccone G, Herbst RS, Manegold C, et al. Gefitinib in combination with gemcitabine and cisplatin in advanced non-small-cell lung cancer: a phase III trial - INTACT 1. J Clin Oncol 2004; 22: 777-784.

36 Herbst RS, Giaccone G, Schiller JH, et al. Gefitinib in combination with paclitaxel and carboplatin in advanced non-small-cell lung cancer: a phase III trial - INTACT 2. J Clin Oncol 2004; 22: 785-794.
37 Kim ES, Hirsch V, Mok T, et al. Gefitinib versus docetaxel in previously treated non-small-cell lung cancer (INTEREST): a randomised phase III trial. Lancet 2008; 372: 1809-1818.

38 Douillard JY, Shepherd F, Hirsch V, et al. Molecular predictors of outcome with gefitinib and docetaxel in previously treated nonsmall-cell lung cancer: data from the randomized phase III INTEREST trial. J Clin Oncol 2010; 28: 744-752.

39 Mok TS, Wu Y-L, Thongprasert S, et al. Gefitinib or carboplatinpaclitaxel in pulmonary adenocarcinoma. N Engl J Med 2009; 361: 947-957.

40 Douillard JY, Kim ES, Hirsh V, et al. Gefitinib (IRESSA) versus docetaxel in patients with locally advanced or metastatic non-small cell lung cancer pre-treated with platinum-based chemotherapy: a randomized, open-label phase III study (INTEREST). J Thoracic Oncol 2007; 2: PRS-02.

41 Cufer T, Vrdoljak E, Gaafar R, et al. Phase II, open-label, randomized study (SIGN) of single-agent gefitinib (IRESSA) or docetaxel as second-line therapy in patients with advanced (stage IIIb or IV) non-small-cell lung cancer. Anticancer Drugs 2006; 17: 401-409.

42 Maruyama R, Nishiwaki Y, Tamura T, et al. Phase III study, V-1532 , of gefitinib versus docetaxel in previously treated Japanese patients with non-small-cell lung cancer. J Clin Oncol 2008; 26: 4244-4252.

43 Lee DH, Park K, Kim JH, et al. Randomized phase III trial of gefitinib versus docetaxel in non-small cell lung cancer patients who have previously received platinum-based chemotherapy. Clin Cancer Res 2010; 16: 1307-1314.

44 Mitsudomi T, Morita S, Yatabe Y, et al. Gefitinib versus cisplatin plus docetaxel in patients with non-small-cell lung cancer harbouring mutations of the epidermal growth factor receptor (WJTOG3405): an open label, randomised phase 3 trial. Lancet Oncol 2010; 11: 121-128.

45 Maemondo M, Inoue A, Kobayashi K, et al. Gefitinib or chemotherapy for non-small-cell lung cancer with mutated EGFR. N Engl J Med 2010; 362: 2380-2388.

46 Broglio KR, Berry DA. Detecting an overall survival benefit that is derived from progression-free survival. J Natl Cancer Inst 2009; 101: $1642-1649$.

47 Fukuoka M, Wu Y, Thongprasert S, et al. Biomarker analyses from a phase III, randomized, open-label, first-line study of gefitinib (G) versus carboplatin/paclitaxel $(\mathrm{C} / \mathrm{P})$ in clinically selected patients (pts) with advanced non-small cell lung cancer (NSCLC) in Asia (IPASS). J Clin Oncol 2009; 27: Suppl. 15, 8006.

48 Kris M, Mok T, Kim E, et al. Response and progression-free survival in 1006 patients with known EGFR mutation status in phase III randomized trials of gefitinib in individuals with nonsmall cell lung cancer. Eur J Cancer 2009; 45: Suppl. 1, O-9003.

49 Cortes-Funes H, Gomez C, Rosell R, et al. Epidermal growth factor receptor activating mutations in Spanish gefitinib-treated nonsmall-cell lung cancer patients. Ann Oncol 2005; 16: 1081-1086.

50 Rosell R, Moran T, Queralt C, et al. Screening for epidermal growth factor receptor mutations in lung cancer. N Engl J Med 2009; 361: 958-967.

51 Tokumo M, Toyooka S, Kiura K, et al. The relationship between epidermal growth factor receptor mutations and clinicopathologic features in non-small cell lung cancers. Clin Cancer Res 2005; 11: 1167-1173.

52 Yoshida K, Yatabe Y, Park JY, et al. Prospective validation for prediction of gefitinib sensitivity by epidermal growth factor receptor gene mutation in patients with non-small cell lung cancer. J Thorac Oncol 2007; 2: 22-28.

53 Reck M. A major step towards individualized therapy of lung cancer with gefitinib: the IPASS trial and beyond. Expert Rev Anticancer Ther 2010; 10: 955-965.

54 Weinstein IB, Joe A. Oncogene addiction. Cancer Res 2008; 68: 3077-3080. 